\title{
Nota per le fonti della persecuzione anticristiana di Nerone e le sue conseguenze, alla luce di due recenti apporti critici
}

\author{
ILARIA RAMELLI
}

\section{RIASSUNTO}

L'articolo analizza le testimonianze di Tacito, di Clemente Romano e di

Giovenale sulla persecuzione anticristiana di nerone e presenta

la scoperta di una delle piu antiche lettere cristiane extracanoniche (POxy XLII 3057).

\section{SUMMARY}

The article analyses the evidences of Tacitus, Clemens of Rome and Juvenal about the neronian anti-cristian persecution and presents the recent discovery of one of the most ancient Christian Letters (POxy XLI/ 3057).

In un recente articolo Carlo Pellegrino ${ }^{1}$ ha proposto una interessante -e, a mio parere, risolutiva- emendazione al testo di Tac. Ann. XV 44, 4, in cui lo storico latino tratta della violenta quanto spettacolare persecuzione anticristiana scatenata da Nerone a Roma nel 64. Sarà opportuno citare per esteso il passo in questione, secondo la proposta critica dello studioso:

ergo abolendo rumori Nero subdidit reos et quaesitissimis poenis affecit quos per flagitia invisos vulgus Christianos appellabat [...] igitur primum correpti qui fatebantur, deinde indicio eorum multitudo ingens haud proinde in crimine incendii quam odio humani generis convicti sunt. Et pereuntibus

1 C. Pellegrino, «Una crux tacitiana: Ann. XV, 44, 7», Latomus 59 (2001), pp. 105-108. 
addita ludibria, ut ferarum tergis contecti laniatu canum interirent, aut crucibus adfixi [aut] flammandi atque, ubi defecisset dies, in usum nocturni luminis uterentur. Hortos suos ei spectaculo Nero obtulerat et circense ludicrum edebat, habitu aurigae, permixtus plebi, vel curriculo insistens. Unde quamquam adversus sontes et novissima exempla meritos miseratio oriebatur.

Nella frase su cui l'Autore concentra la propria attenzione, il codice Mediceus alter ${ }^{2}$ legge: «et pereuntibus addita ludibria, ut ferarum tergis contecti laniatu canum interirent, aut crucibus adfixi aut (in)flammandi ${ }^{3}$ atque ubi defecisset dies in usum nocturni luminis urerentur". Di fronte a questo testo, il Pellegrino assume una posizione mediana tra quanti editori accettano il testo tràdito ${ }^{4} \mathrm{e}$ quanti invece vi intervengono assai pesantemente, o espungendo addirittura le parole «aut crucibus adfixi aut flammandi» ${ }^{5}$, o ponendole tra cruces ${ }^{6}$, oppure elimimando "aut flammandi atque" ${ }^{7} \mathrm{O}$ ancora emendando quest'ultima espressione in varî modi ${ }^{8}$. Criticando le ricostruzioni precedenti, in quanto non avrebbero còlto il senso del passo, egli propone di espungere aut da aut crucibus adfixi [aut] flammandi. Con questo semplice e unico intervento, a differenza di quanto si può evincere dalle precedenti letture, risulta che il supplizio della croce e quello dell'essere arsi non erano applicati a gruppi di persone differenti, bensi alle stesse: in questo senso aveva visto giusto $\mathrm{K}$. Wellesley, il quale, eliminando aut e sostituendolo con ut ("aut crucibus adfixi ut flammandi»), aveva inteso che fossero applicati alle stesse persone, in tempi diversi, i due supplizi, come egli stesso spiega nella Appendix critica della sua edizione teubneriana: "quomodo infelices isti novo excogitato supplicio primum tunica molesta induti tum crucibus sublati postremo vespere flammati per Neronis hortos et in circo nocturnum lumen morientes ex alto praeberent» (p. 159). I Cristiani, dunque, non sarebbero stati, alcuni, crocifissi e, altri, dati alle fiamme, bensi, al contempo, sia crocifissi sia arsi. Giustamente il Pellegrino

\footnotetext{
2 M2, Laurentianus LXVIII, 2, della metà dell'XI secolo.

3 In $\mathrm{M} 2$ in- è soprascritta da una mano molto posteriore all'altezza della lettera $f$ di flammandi.

4 Es. Jacob, Furneaux e Pitman.

Come Nipperdey, Büchner, Beaujeu e Draeger.

Cosi Jackson.

Cosi Andresen, Goelzer, Heubner, Hanslik, Römer e Wuilleumier.

8 C. Halm propone: "aut flamma usti, alique ubi defecisset dies"; E. Koestermann e A. Arici: «atque flammati, ubi defecisset dies"; K. Wellesley: "aut crucibus adfixi, ut flammandi, ubi dies defecisset".
} 
insiste sul carattere derisorio e spettacolare del supplizio, sul fatto che Tacito mira a porre in luce «l'aspetto di scherno e irrisione che conferiva alla morte di costoro un effetto scenico di sicura efficacia" 9 ; con questa semplice emendazione "ottiene il dovuto risalto, sul supplizio in sé e per sé, il tragico scherno al medesimo connesso" ${ }^{10}$.

Un altro elemento a mio avviso molto interessante dell'intervento del Pellegrino consiste nell'accostamento al testo tacitiano cosi emendato di tre versi della prima Satira di Giovenale (vv. 155-157): "pone Tigellinum: taeda lucebis in illa / qua stantes ardent qui fixo pectore fumant, / et latum media sulcum deducis harena". $E$ evidente che con Tacito l'immagine tratteggiata ha in comune sia il tipo di supplizio -essere crocifissi e ardere come una fiaccola - sia il contesto del circense ludicrum e la crudele spettacolarità del supplizio.

Lo avevo già proposto di leggere nel passo di Giovenale una chiara rievocazione delle spettacolari esecuzioni dei Cristiani a Roma dopo l'incendio del 64, ponendo in stretta connessione il paso di Tacito e quello di Giovenale, in un articolo su Espacio. Tiempo y Forma dedicato a crux negli autori latini di i-ll secolo, e, più brevemente, in un altro su Gerión incentrato su un'ipotesi di possibili allusioni al Cristianesimo in Giovenale ${ }^{11}$. In effetti, l'accenno di Giovenale ai condannati a morte bruciati come torce umane e uccisi nell'arena del circo ai tempi di Tigellino si richiama ai supplizi spettacolari che furono inflitti ai presunti incendiarî durante la persecuzione neroniana nel 64, quando Tigellino affiancava Nerone: anche in Tacito sono espliciti gli accenni all'uso dei condannati come torce umane e al circense ludicrum voluto da Nerone: accettando, poi, la congettura di Pellegrino,

9 Pellegrino, «Una crux», p. 106.

10 Pellegrino, “Una crux», p. 108; Pellegrino spiega: "quei disgraziati venivano non soltanto posti in croce, ma preparati per essere accesi, presumibilmente con il corpo ricoperto di pece ovvero rivestiti della tunica molesta, e servire pertanto da torce viventi al calare della sera per illuminare i giardini dell'imperatore offerti, dalio stesso, come sede per l'iconsueto spettacolo, mentre al contempo, sempre Nerone, circense hudicrum edebat, habitu aurigae permixtus plebi vel curriculo insistens".

"Alcune osservazioni sulle occorrenze di crux in Manilio, Seneca, Giovenale e Marziale», Espacio, Tiempo y Forma, ser. II, 12 (1999), pp. 241-252; “La Satira IV di Giovenale e it supplizio di san Giovanni a Roma sotto Domiziano", Gerión 18 (2000), pp. 343-359. Ovviamente, la vicinanza cronologica delle nostre pubblicazioni ha impedito al Pellegrino di avere notizia dei miei lavori: per questo egli a p. 108 dichiara di non essere a conoscenza di nessuno che abbia posto in relazione tra loro i due passi, tranne Furneaux nel suo commento a

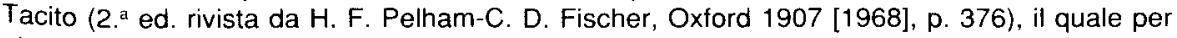
altro non trae nessuna conseguenza. 
diviene ancora più chiaro e stringente il parallelismo tra i due autori, che sembrano proprio riferirsi allo stesso, preciso episodio.

Le fonti parallele per la persecuzione neroniana ci confermano in questa tesi. Clemente Romano, scrivendo ai Corinzi alla fine del I secolo, ricorda, come Tacito e come Giovenale, la spettacolarità delle esecuzioni dei Cristiani durante la persecuzione neroniana ${ }^{12}:$ «per gelosia [ $\left.\zeta \hat{\eta} \lambda \circ \zeta\right]$ donne, <giovinette e fanciulle>, sotto le spoglie Dirci e di Danaidi, furono perseguitate e soffirono oltraggi terribili ed empî per la fede» $(6,2){ }^{13}$. Similmente, se Seneca appare in effetti impressionato in modo particolare dall'apparato spettacolare degli strumenti di morte, fra cui specialmente la stessa croce, soprattutto nelle Epistulae ad Lucilium, degli anni successivi al ritiro politico (62-64 d.C.) ${ }^{14}$, anche Marziale ${ }^{15}$ sembra ricordare con particolare impressione la spettacolarizzazione del supplizio della croce, tipica degli episodî neroniani: egli, che nel 64 era a Roma, appoggiato dalla famiglia di Seneca e frequentatore dei circoli dell'opposizione senatoria a Nerone, menziona un condannato crocifisso veramente, sulla scena del mimo Laureolus: uno dei capi d'accusa, come per i Cristiani nel 64 , era quello di avere incendiato Roma ("subdiderat saevas vel tibi, Roma, faces»). Infine, anche Lucano, il quale scrive il X libro del Bellum civile o Pharsalia proprio a Roma nel 64-65, cita un supplizio spettacolare e molto simile - fungere da torcia umana sospesi in croce-: «crucibus flammisque lucemus" (X 365) ${ }^{16}$. Anche qui non è escluso che Lucano avesse presenti gli episodî persecutorî di Nerone.

Queste, dunque, possono essere considerate, almeno per la maggior parte, fonti per la persecuzione anticristiana di Nerone. II tema della crocifissione in relazione ai Cristiani è poi ben presente in un autore contemporaneo agli eventi e facente parte del circolo neroniano nel 64: Petronio; nel suo Satyricon, nell'episodio dei crocifissi che conclude la novella della Matrona di Efeso, forse si può leggere addirittura una parodia della morte in croce e della

12 Clem. Rom. ad Cor. 1, 1: «improvvise disgrazie [ $\alpha \grave{\phi} \phi v i \delta i ́ o v \varsigma \sigma v \mu \phi o \rho \alpha ́ \varsigma]$ ed avversità capitateci l'una dietro l'altra".

${ }_{13}$ Lettura incerta: cfr. Die Apostolischen Väter, edd. F. X. Funk-K. Bihlmeyer-M. Whittaker;

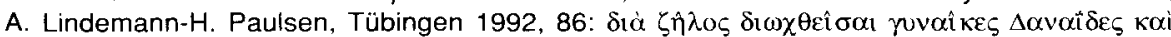

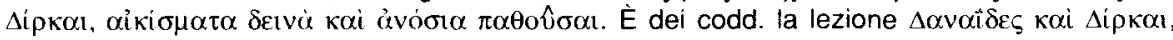
mentre Wordsworth ha proposto ve $\alpha$ vi $\delta \varepsilon \varsigma \pi \alpha i \delta i \sigma \kappa \alpha l$ (si veda l'apparato critico).

14 Ep. 14, 4-6, 101, 10-14.

15 Spect. 7.

16 Parlano i cortgiani egiziani Achilla e Potino. 
resurrezione di Gesù Cristo; la crocifissione, unitamente a polemiche anticristiane legate alla accusa di $\tau v \mu \beta \omega \rho v \chi i \alpha$, si ritrova poi in un contemporaneo di Petronio, Caritone di Afrodisia, e non manca nemmeno nell'altro romanziere latino, Apuleio, per divenire anzi pressoché topico nei romanzieri antichi successivi, per esempio in Senofonte Efesio ${ }^{17}$. La comparsa di questa tematica in concomitanza cronologica e polemica con la nascita del Cristianesimo induce pure a qualche riflessione.

Tutte queste osservazioni, insomma, confortano la supposizione che sia Tacito sia Giovenale, come del resto anche altri autori, avessero presente la persecuzione neroniana a Roma e vi facessero esplicito riferimento: molti, in effetti, erano rimasti colpiti dalla crudeltà spettacolare di quelle uccisioni, come attesta Tacito stesso, il quale parla di miseratio tra gli astanti (Ann. XV 44). È certo, quindi, che Giovenale conoscesse i Cristiani: e questo dà più valore alla mia ipotesi sul possibile riferimento al supplizio di Giovanni a Roma nella Satira IV del poeta latino: nella storia dell'enorme pesce straniero che sarebbe res fiscie che, pescato presso un luogo pieno di delatori, viene condotto al cospetto di Domiziano -che lo giudica per un delitto religioso, nelle vesti di pontifex maximus, pretendendo di avere una dis aequa potestas - per essere gettato in un profondo recipiente e arrostito, ho proposto ${ }^{18}$, infatti, di leggere una serie di allusioni all'arresto di s. Giovanni evangelista a Roma sotto Domiziano - in un'epoca di delazioni diffuse a carico dei Cristiani e per il fiscus ludaicus - e alla sua immersione in un recipiente pieno di olio bollente, come attestano Tertulliano (Praescr. Haer. 36, 2-3: in oleum igneum demersus) e Gerolano (in dolium ferventis olei, In Matth. III 20, 23; Adv. lovin. I 26). Che Giovenale avesse presenti i Cristiani perseguitati non solo sotto Nerone, ma anche sotto Domiziano (il "calvo Nerone" del v. 38 = il «mezzo Nerone" di Tert. Apol. 5, 5), sembra attestato anche dalla critica che egli rivolge a Domiziano stesso, nella

17 Si veda il mio /l romanzo antico e il Cristianesimo: contesto e contatti, Madrid 2001, GraecoRomanae Religionis Electa Collectio, 6, capp. I per Caritone, II per Senofonte, VIII per Petronio, IX per Apuleio e passim per la topica nel romanzo antico in generale. Per la data di Caritone rinvio, con amplia documentazione, allo stesso capitolo I: aggiungo qui un articolo recente, E. P. Cueva, "The Date of Chariton's Chaereas and Callirhoe Revisited", Classica et Mediaevalia. Revue danoise de philologie et d'historie 51 (2000), pp. 197-208, il quale propone una lieve postdatazione rispetto a quella corrente, che comunque non inficia minimamente il mio discorso. Cfr. oggi il mio "Indizi della conoscenza del Nuovo Testamento nei romanzi antichi" in $Y$ l contributo delle scienze storiche allinterpretazione del Nuovo Testamento, Atti del Congresso Internazionale a cura del Pontificio Comitato di Scienze Storiche (Roma, 2-6 ottobre 2002), in corso di pubblicazione.

18 "La Satira IV di Giovenale", cit., passim. 
medesima Satira IV, per avere mandato a morte il senatore M'. Acilio Glabrione, il quale era appunto cristiano ${ }^{19}$.

I Cristiani, adoratori di un crocifisso - cosa che i pagani sapevano molto bene, come dimostra il graffito del Palatino del "I secolo, in cui un Cristiano è ritrato in atto di adorazione di un crocifisso con testa d'asino ${ }^{20}$ -e accusati in quell'occasione di essere gli incendiarî, forse per una sorta di contrappasso furono allora sia crocifissi sia bruciati.

La persecuzione di Nerone e, poi, quella di Domiziano, a causa dello statuto del Cristianesimo come superstitio illicita - che comportava la condanna a morte di chi, denunciato come Cristiano, perseverasse nella sua fede - ${ }^{21}$, imposero ai Cristiani uno stato di clandestinità che perdurò almeno fino all'età severiana e che si esplicò nell'astensione dalle cariche pubbliche, nell'impiego di formule criptate e in una forte circospezione nell'esporsi e nel farsi riconoscere - per questo, fra l'altro, pochissime epigrafi cristiane sono riconoscibili come tali- ${ }^{22}$. Una chiara descrizione della situazione di timore e circospezione in cui vivevano le comunità cristiane ci è offerta da una lettera su papiro che, secondo quanto proponiamo Orsolina Montevecchi $e$ io in un recente articolo su Aegyptus ${ }^{23}$, sembra essere tra la più antiche lettere cristiane esistenti, al di fuori del Canone biblico: sarebbe, in particolare, il primo documento cristiano che ci rimanga in Egitto. Si tratta di POxy XLII $3057^{24}$, una lettera molto ben scritta e curata, inviata tra la fine del I e gli inizi del II secolo da

\footnotetext{
19 Rinvio ancora senz'altro a «La Satira IV di Giovenale» e anche al mio "L'omen per Acilio Glabrione e per Traiano", Rivista di Storia della Chiesa in Italia, 55 (2001), pp. 389-394.

20 Documentazione e bibliografia nel mio / romanzi antichi e il Cristianesimo: contesto e contatti, cit., cap. IX.

21 Secondo M. Sord, I Cristiani e I'Impero Romano, Milano 1984, pp. 25-26, il Cristianesimo sarebbe stato dichiarato superstitio illicita già nel 35 , con il senatoconsulto che rispondeva negativamente alla proposta tiberiana di consecratio di Cristo, secondo Tertulliano (Apol. 5, 2).

22 Ho studiato questa situazione in "Cristianesimo e vita politica. Il cripto-cristianesimo nelle classi dirigenti romane nei primi due secoli», in corso di stampa in Aevum.

23 I. RAMELLI, «Una delle più antiche lettere cristiane extracanoniche?», Aegyptus 80 (2000)[2002], pp. 169-188; O. MONTEVECCH, ibid., pp. 189 sig.

24 Cfr. O. Montevecchl, Aegyptus 55 (1975), p. 302; C. J. Hemer, "Ammonius to Apollonius: Greetings", Buried History 12 (1976), pp. 84-91; P. J. PARSONS, “The Earliest Christian Letter?", in Miscellanea Papyrologica (Pap. Flor. VII), Firenze 1980, p. 289; G. R. Stanton, "The Proposed Earliest Christian Letter on Papyrus and the Origin of the Term Philallelia", ZPE 54 (1984), pp. 49-63; S. R. LleWELYN-R. A. KeARSLEY, New Documents Illustrating Early Christianity, VI: $A$ Review of the Greek Inscriptions and Papyri published in 1980-81, Macquaire University 1992, pp. $169-177$
} 
un certo Ammonio a un tale Apollonio. Sono due privati, i quali però lasciano intravvedere alle loro spalle una comunità di cristiani ${ }^{25}$, rivelata anche dalla presenza di una evidente alternanza di singolare e plurale sia nella prima che nella seconda persona, riscontrabile anche in altri papiri cristiani epistolari ${ }^{26}$. In particolare, attraverso Apollonio, Ammonio si rivolge alla "comunità del suo "fratello»: «non voglio, infatti, che voi, fratelli, facciate alcuna diffenza tra me e un altro. lo prego infatti che rimangano tra di voi concordia e amore reciproco, perché non siate oggetto di voci malevole, e non vi accada come a noi. Infatti, l'esperienza mi induce a spronarvi a starvene in pace e a non dare occasione ad altri contro di voi's 27. Come si nota da queste parole, inoltre, Ammonio non appare preoccupato soltanto per la concordia della comunità - preoccupazione che compare anche nelle lettere neotestamentarie-, ma anche per l'ostilità che evidentemente regna intorno alla sua comunitá, come intorno a quella di Ammonio: in questa situazione, eventuali dissidî interni alla comunità favorirebbero questa ostilità dall'esterno.

In effetti, tra I e $\|$ secolo, il Cristianesimo era ufficialmente superstitio illicita e su di esso gravavano accuse di flagitia, come attesta lo stesso Tacito (Ann. XV 44): sul finire del I secolo ci fu la persecuzione di Domiziano, quindi la pace di Nerva e, subito dopo, il rescritto di Traiano

25 Riporto il testo (tr. mia): “Ammonio saluta il fratello Apollonio. Ho ricevuto la lettera contrassegnata dal x (kechiasméne), il porta-mantello, i mantelli da viaggio, e le zampogne di poco pregio. $\mathrm{E}$, in quanto ai mantelli da viaggio, non li ho ricevuti come vecchi, bensì, se possibile, migliori dei nuovi, in virtù della tua intenzione (prohairesis). Non voglio, però, a fratello, che tu mi opprima con le tue continue gentilezze (philanthropiai) [...] dato che non sono in grado di ricambiare: riteniamo di averti offerto solo questo: l'intenzione (prohairesis) di dimostrare il nostro affetto (diáthesis philiké). D'altra parte, ti esorto, o fratello, a non darti più pensiero per la chiave del monolocale: non voglio, infatti, che voi, fratelli, facciate alcuna differenza tra me e un altro. lo prego, infatti, che rimangano tra voi concordia e amore reciproco (homónoia, philallelia), perché non siate oggetto di voci malevole, e non vi succeda come a noi. Infatti, l'esperienza mi induce a spronarvi a starvene in pace e a non dare occasione ad altri contro di voi. Tenta, dunque, di fare questo anche per amor mio, facendomi una cosa grata (charisámenos) in quello che tu riconosci senz'altro come buono (agathón). Se riceverai tutta la lana da Salvio, e ti sarà gradita, scrivimelo in risposta; nella lettera precedente, infatti, ti ho scritto cose ridicole, che ammetterai. Poiché la mia anima si rasserena, ogni qual volta sia presente il tuo nome, e questo, benché non sia abituata a rimanere tranquilla, a causa di quanto sta accadendo, ma sopporta (hypophérei). lo, Leonas, ti saluto, o padrone (déspota), te e tutti i tuoi. Sta'bene, o onorevolissimo (timiótate). [Sul verso:] Ad Apollonio, figlio di Apollo (?), ispettore fratello".

${ }^{26}$ H. I. BELL, Jews and the Christians in Egypt, Westport 1924, 1972 rist., p. 76; LLEWELYN, New Documents ... VI, p. 170.

27 Che, del resto, ci si indirizzi all'intera «comunità» di Apollonio risulta chiaro dalla formula di saluto finale: «Ti saluto... te e tutti i tuoi". 
per cui i Cristiani conquirendi non sunt, ma, se denunciati, dovevano essere messi sotto processo e, se perseverantes, condannati a morte. Ora, tali denunce erano sporte da privati ostili: quindi i Cristiani dovevano cercare di passare il più possibile inosservati, di non dare occasione di ostilità e malevolenza. E Ammonio raccomanda appunto questo, perché ha già avuto esperienza, nella sua comunità, di questi fatti spiacevoli («non siate simili a noi ... l'esperienza mi induce a spronarvi a stare in pace e non dare occasione ad altri contro di voi»). Perciò egli torna con insistenza su questa situazione di pericolo e di ostilità, che gli causa gravi angustie, ancora alla fine della lettera: «La mia anima, infatti, si rasserena, ogni qual volta sia presente il tuo nome, e questo benché non sia abituata a stare tranquilla, a causa di quanto sta accadendo... ma sopporta». Non è escluso che nella comunità di Ammonio ci siano state denunce contro i Cristiani. Del resto, un analogo contesto di lotte interne e di attacchi persecutorii dall'esterno e l'accenno alle gravi vicissitudini presenti, si trova nelle lettere di Clemente Romano ai Corinzi, che riportano proprio ai fatti di questo periodo, con le persecuzioni di Nerone e di Domiziano: ed è proprio Clemente, come accennavamo, a ricordare i supplizi spettacolari delle donne cristiane costrette a scendere nell'arena sotto le spoglie di Dirci e di Danaidi: quel tipo di persecuzione che Nerone trasformò anche in uno spettacolo crudele, tale da suscitare miseratio perfino tra gli spettatori pagani (Ann. XV 44). Nella lettera Ammonio, oltre a chiamare «fratelli » Apollonio e $\mathrm{i}$ suoi, e a fare emergere valori quali umiltà, amore reciproco, amicizia, concordia e simili -il lessico della $\phi i \lambda i \alpha$

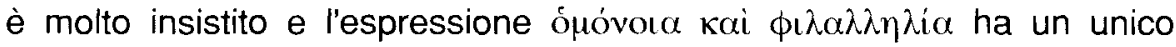
parallelo, e solo nella letteratura cristiana ${ }^{28}$ - lascia come segno del suo Cristianesimo un X (l'iniziale della formula di saluto $\chi \alpha \dot{\alpha} \iota \rho \varepsilon v$ ) sopralineato: secondo Orsolina Montevecchi ${ }^{29}$, si tratta di un nomen sacrum, di una abbreviazione di Christós: proprio in quel periodo, infatti, si stava diffondendo l'uso dei nomina sacra. Evidentemente i due, scambiando la loro corrispondenza, contrassegnavano in questo modo le loro lettere: per questo Ammonio, parlando di una lettera precedente di Apollonio, dice che essa era "contrassegnata con il $X$ » ( $\kappa \varepsilon \chi 1 \alpha \sigma \mu \varepsilon \dot{v} \eta)$. Se si pensa, quindi, all'ostilità gravante contro le comunità di Ammonio e di Apollonio, si comprende quest'abitudine, certo imposta dallo stato di clandestinità in cui i Cristiani si trovavano, di usare formule criptate, che potevano essere

\footnotetext{
28 Ramelli, "Una delle piú antiche lettere», cit.

29 Art. cit.
} 
confuse con altre già usitate in ambito pagano ${ }^{30}$ : tale sembra la lettera $X$ sopralineata, che agli occhi di un pagano non avrebbe destato sospetti, in quanto sarebbe stata solo l'iniziale del verbo $\chi \alpha i \rho \varepsilon ı v$, mentre per un Cristiano era il segno di Cristo stesso. Quindi, la lettera di Ammonio, se è cristiana, costituisce un'attestazione delle difficoltà incontrate dai Cristiani sotto Traiano. Le premesse di questa situazione erano state poste, comunque, da Nerone, il quale per primo aveva posto in atto, con una persecuzione aperta, le conseguenze implicite nella dichiarazione del Cristianesimo come superstitio illicita ${ }^{31}$.

30 Si pensi al caso dei collegia che nascondevano vere e proprie chiese. Si può vedere, con bibliografía, RAMELLI, "Cristiani e vita politica», cit.

31 Sord, I Cristiani, pp. 37-43. 